\title{
Hemophagocytic Lymphohistiocytosis: Early Treatment Leading to an Excellent Outcome
}

\author{
Gerald Marín-García, MD; José María-Rios, MD; and William Rodriguez-Cintrón, MD
}

$\mathrm{HLH}$ is a rare and deadly disease increasingly more present in adults, but following treatment protocol may yield favorable results.

Gerald Marín-García is an
Emergency Medicine-Critical
Care Attending Physician,
and William Rodriguez-
Cintrón is Chairperson
Pulmonary-Critical Care
Department, Pulmonary-
Critical Care Fellowship
Program Director, both at
Veterans Affairs Caribbean
Healthcare System in San
Juan, Puerto Rico. José
María-Rios is Critical Care
Fellow at AdventHealth in
Orlando, Florida.
Correspondence:
William Rodriguez-Cintrón
(william.rodriguez@va.gov)

Fed Pract. 2021;38.

Published online

September 9 .

doi:10.12788/fp.0162
$\mathrm{H}$ emophagocytic lymphohistiocytosis $(\mathrm{HLH})$ is a rare and deadly disease in which unregulated proliferation of histiocytes and T-cell infiltration takes place. It is known as a pediatric disease in which gene defects result in impaired cytotoxic NK- and T-cell function. It has been associated with autosomal recessive inheritance pattern. Without therapy, survival for these patients with active familial HLH is approximately 2 months.

Recognition of the disease has increased over the years, and as a result the diagnosis of $\mathrm{HLH}$ in adults also has increased. An acquired form can be triggered by viruses like Epstein-Barr virus, influenza, HIV, lymphoid malignancies, rheumatologic disorders, or immunodeficiency disorders. Survival rates for untreated $\mathrm{HLH}$ have been reported at $<5 \%$. $^{1}$ Despite early recognition and adequate treatment, HLH carries an overall mortality of $50 \%$ in the initial presentation, $90 \%$ die in the first 8 weeks of treatment due to uncontrolled disease. ${ }^{2}$

\section{CASE PRESENTATION}

A 56-year-old man with no active medical issues except for a remote history of non-Hodgkin lymphoma treated with chemotherapy and splenectomy in 1990 presented to the Veterans Affairs Caribbean Healthcare System in San Juan, Puerto Rico. He was admitted to the medicine ward due to community acquired pneumonia. Three days into admission his clinical status deteriorated, and the patient was transferred to the intensive care unit (ICU) due to acute respiratory failure and sepsis secondary to worsening pneumonia. Chest imaging demonstrated rapidly progressing diffuse bilateral infiltrates. Due to the severity of the chest imaging, a diagnostic bronchoscopy was performed.

The patient's antibiotics regimen was empirically escalated to vancomycin $1500 \mathrm{mg}$ IV every 12 hours and meropenem $2 \mathrm{~g}$ IV every 8 hours. Despite optimization of therapy, the patient did not show clinical signs of improvement. Febrile episodes persisted, pulmonary infiltrates and hypoxemia worsened, and the patient required a neuromuscular blockade. Since the bronchoscopy was nondiagnostic and deterioration persistent, the differential diagnosis was broadened. This led to the ordering of inflammatory markers. Laboratory testing showed ferritin levels $>16,000 \mathrm{ng} / \mathrm{mL}$, pointing to $\mathrm{HLH}$ as a possible diagnosis. Further workup was remarkable for triglycerides of $1234 \mathrm{mg} / \mathrm{dL}$ and a fibrinogen of $0.77 \mathrm{~g} / \mathrm{L}$. In the setting of bicytopenia and persistent fever, HLH-94 regimen was started with dexamethasone $40 \mathrm{mg}$ daily and etoposide $100 \mathrm{mg} / \mathrm{m}^{2}$. CD25 levels of $154,701 \mathrm{pg} / \mathrm{mL}$ were demonstrated as well as a decreased immunoglobulin (lg) G levels with absent $\lg \mathrm{M}$ and $\lg \mathrm{A}$. Bone marrow biopsy was consistent with hemophagocytosis. The patient eventually was extubated and sent to the oncology ward to continue chemotherapy.

\section{DISCUSSION}

A high clinical suspicion is warranted for rapid diagnosis and treatment as HLH evolves in most cases to multiorgan failure and death. The diagnostic criteria for HLH was developed by the Histiocyte Society in 1991 and then restructured in

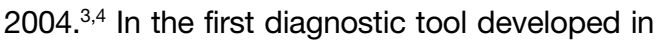
1991, diagnosis was based on 5 criteria (fever, splenomegaly, bicytopenia, hypertriglyceridemia and/or hypofibrinogenemia, and hemophagocytosis). Three additional laboratory findings were also described as part of HLH diagnosis since 2004: low or absent NK-cell-activity, hyperferritinemia of $>500 \mathrm{ng} / \mathrm{dL}$, and high-soluble interleukin-2-receptor levels (CD25) > $2400 \mathrm{U} /$ $\mathrm{mL}$. Overall, 5 of 8 criteria are needed for the HLH diagnosis.

Despite the common use of these diagnostic criteria, they were developed for the pediatric population but have not been validated for adult patients. ${ }^{5}$ For adult patients, the HScore was developed in 2014. It has 9 variables: 3 are based on clinical findings (known underlying immunosuppression, high temperature, and organomegaly; 5 are based on laboratory values 
(ferritin, serum glutamic oxaloacetic transaminase, cytopenia, triglycerides, and fibrinogen levels); the last variable uses cytologic findings in the bone marrow. In the initial study, probability of having $\mathrm{HLH}$ ranged from $<1 \%$ with an $\mathrm{HScore}$ of $\leq 90 \%$ to $>99 \%$ with an HScore of $\geq 250$ in noncritically ill adults. ${ }^{5}$ A recently published retrospective study demonstrated the diagnostic reliability of both the HLH-2004 criteria and HScore in critically ill adult patients. This study concluded that the best prediction accuracy of HLH diagnosis for a cutoff of 4 fulfilled HLH-2004 criteria had a $95.0 \%$ sensitivity and $93.6 \%$ specificity and HScore cutoff of 168 reached a $100 \%$ sensitivity and $94.1 \%$ specificity. $^{6}$

The early negative bronchoscopy lowered the possibility of an infection as the etiology of the clinical presentation and narrowed the hyperferritinemia differential diagnosis. Hyperferritinemia has a sensitivity and specificity of $>90 \%$ for diagnosis when above $10,000 \mathrm{ng} / \mathrm{dL}$ in the pediatric population. ${ }^{7}$ This is not the case in adults. Hyperferritinemia is a marker of different inflammatory responses, such as histoplasmosis infection, malignancy, or iron overload rather than an isolated diagnostic tool for $\mathrm{HLH}^{8}{ }^{8}$ It has been reported that CD25 levels less than the diagnostic threshold of $2400 \mathrm{U} / \mathrm{mL}$ have a $100 \%$ sensitivity for the diagnosis and therefore can rule out the diagnosis. When this is taken into consideration, it can be concluded that CD25 level is a better diagnostic tool when compared with ferritin, but its main limitation is its lack of widespread availability. ${ }^{9}$ Still, there is a limited number of pathologies that are associated with marked hyperferritinemia, specifically using thresholds of more than $6000 \mathrm{ng} / \mathrm{dL} .{ }^{10}$ Taking into consideration the high mortality of untreated $\mathrm{HLH}$, isolated hyperferritinemia still warrants HLH workup to aggressively pursue the diagnosis and improve outcomes.

The goal of therapy in HLH is prompt inactivation of the dysregulated inflammation with aggressive immunosuppression. In our deteriorating patient, the treatment was started with only 4 of the $8 \mathrm{HLH}-2004$ diagnostic criteria being met. As per the 2018 Histiocyte Society consensus statement, the decision to start the HLH-94 treatment relies on not only the HLH-2004 diagnostic criteria, but also the patient's clinical evolution. ${ }^{11}$ In 1994 the Histiocyte Society also published a treatment protocol termed HLH-94. A Korean retrospective study demonstrated that this protocol led to a 5-year survival rate of 60 to $80 \%$ depending on the $\mathrm{HLH}$ trigger and response to initial treatment. ${ }^{12}$ The protocol consists of etoposide at $150 \mathrm{mg} / \mathrm{m}^{2}, 2$ weekly doses in the first 2 weeks and then 1 dose weekly for the next 6 weeks. Dexamethasone is the steroid of choice as it readily crosses the blood-brain barrier. Its dosage consists of $10 \mathrm{mg} / \mathrm{m}^{2}$ for the first 2 weeks and then it is halved every 2 weeks until the eighth week of treatment. A slow taper follows to avoid adrenal insufficiency. Once 8 weeks of treatment have been completed, cyclosporine is added to a goal trough of $200 \mathrm{mcg} / \mathrm{dL}$. If there is central nervous system (CNS) involvement, early aggressive treatment with intrathecal methotrexate is indicated if no improvement is noted during initial therapy. ${ }^{11}$

In 2004 the Histiocyte Society restructured the HLH-94 treatment protocol with the aim of presenting a more aggressive treatment strategy. The protocol added cyclosporine to the initial induction therapy, rather than later in the ninth week as HLH-94. Neither the use of cyclosporine nor the HLH-2004 have been demonstrated to be superior to the use of etoposide and dexamethasone alone or in the HLH-94 protocol, respectively. ${ }^{13}$ Cyclosporine is associated with adverse effects (AEs) and may have many contraindications in the acute phase of the disease. Therefore, the HLH-94 protocol is still the recommended regimen. ${ }^{11}$

To assess adequate clinical response, several clinical and laboratory parameters are followed. Clinically, resolution of fever, improvement in hepatosplenomegaly, lymphadenopathy, and mental status can be useful. Laboratories can be used to assess improvement from organ specific damage such as hepatic involvement or cytopenia. The limitation of these diagnostic studies is that they could falsely suggest an inadequate response to treatment due to concomitant infection or medication AEs. Other markers such as ferritin levels, CD25, and NK cell activity levels are more specific to HLH. Out of them, a decreasing ferritin level has the needed specificity and widespread availability for repeated assessment. On the other hand, both CD25 and NK cell activity are readily available only in specialized centers. An initial high ferritin level is a marker for a poor prognosis, and the rate of decline correlates with mortality. Studies have demonstrated that persistently elevated ferritin levels after treatment initiation are associated with worse outcomes. ${ }^{14,15}$ 
Several salvage treatments have been identified in recalcitrant or relapsing disease. In general, chemotherapy needs to be intensified, either by returning to the initial high dosage if recurrence occurs in the weaning phase of treatment or adding other agents if no response was initially achieved. Emapalumab, an interferon $\gamma$ antibody, was approved by the US Food and Drug Administration for the treatment of intractable $\mathrm{HLH}$ after it demonstrated that when added to dexamethasone, it lead to treatment response in 17 out of 27 pediatric patients, with a relatively safe AE profile. ${ }^{16}$ The goal of intensifying chemotherapy is to have the patient tolerate allogenic stem cell transplant, which is clinically indicated in familial HLH, malignancy induced $\mathrm{HLH}$, and recalcitrant cases. In patients who undergo hematopoietic cell transplantation (HCT) there is a tendency to increase survival to $66 \%$ at 5 years. ${ }^{12}$

\section{CONCLUSIONS}

$\mathrm{HLH}$ is a rare and deadly disease increasingly more present in adults. Our patient who initially presented with a sepsis diagnosis was suspected of having a hematologic etiology for his clinical findings due to markedly elevated ferritin levels. In our patient, the HLH-94 treatment protocol was used, yielding favorable results. Given the lack of specific scientific data backing updated protocols such as HLH-2004 and a comparatively favorable safety profile, current guidelines still recommend using the HLH-94 treatment protocol. Decreasing ferritin levels may be used in conjunction with clinical improvement to demonstrate therapeutic response. Persistence of disease despite standard treatment may warrant novel therapies, such as emapalumab or HCT. Physicians need to be wary of an HLH diagnosis as early identification and treatment may improve its otherwise grim prognosis.

\section{Author disclosures}

The authors report no actual or potential conflicts of interest with regard to this article.

\section{Disclaimer}

The opinions expressed herein are those of the authors and do not necessarily reflect those of Federal Practitioner, Frontline Medical Communications Inc., the US Government, or any of its agencies. This article may discuss unlabeled or investigational use of certain drugs. Please review the complete prescribing information for specific drugs or drug combinations-including indications, contraindications, warnings, and adverse effectsbefore administering pharmacologic therapy to patients.

\section{References}

1. Chen TY, Hsu MH, Kuo HC, Sheen JM, Cheng MC, Lin YJ. Outcome analysis of pediatric hemophagocytic lymphohistiocytosis. J Formos Med Assoc. 2021;120(1, pt 1):172-179. doi:10.1016/j.jfma.2020.03.025

2. Henter JI, Samuelsson-Horne A, Aricò M, et al. Treatment of hemophagocytic Iymphohistiocytosis with HLH-94 immunochemotherapy and bone marrow transplantation. Blood. 2002;100(7):2367-2373. doi:10.1182/blood-2002-01-0172

3. Henter Jl, Elinder G, Ost A. Diagnostic guidelines for hemophagocytic lymphohistiocytosis. The FHL Study Group of the Histiocyte Society. Semin Oncol. 1991;18(1):29-33.

4. Henter Jl, Horne A, Aricó M, et al. HLH-2004: Diagnostic and therapeutic guidelines for hemophagocytic lymphohistiocytosis. Pediatr Blood Cancer. 2007;48(2):124-131. doi:10.1002/pbc.21039

5. Knaak C, Nyvit P, Schuster FS, et al. Hemophagocytic lymphohistiocytosis in critically ill patients: diagnostic reliability of HLH-2004 criteria and HScore. Crit Care. 2020;24(1):244. Published 2020 May 24. doi:10.1186/s13054-020-02941-3

6. Fardet L, Galicier L, Lambotte O, et al. Development and validation of the HScore, a score for the diagnosis of reactive hemophagocytic syndrome. Arthritis Rheumatol. 2014;66(9):2613-2620. doi:10.1002/art.38690

7. La Rosée P, Horne A, Hines M, et al. Recommendations for the management of hemophagocytic lymphohistiocytosis in adults. Blood. 2019;133(23):2465-2477. doi:10.1182/blood.2018894618

8. Schaffner M, Rosenstein L, Ballas Z, Suneja M. Significance of Hyperferritinemia in Hospitalized Adults. Am J Med Sci. 2017;354(2):152-158. doi:10.1016/j.amjms.2017.04.016

9. Hayden A, Lin M, Park S, et al. Soluble interleukin-2 receptor is a sensitive diagnostic test in adult HLH. Blood Adv. 2017;1(26):2529-2534. Published 2017 Dec 6. doi:10.1182/bloodadvances.2017012310

10. Belfeki N, Strazzulla A, Picque M, Diamantis S. Extreme hyperferritinemia: etiological spectrum and impact on prognosis. Reumatismo. 2020;71(4):199-202. Published 2020 Jan 28. doi:10.4081/reumatismo.2019.1221

11. Ehl S, Astigarraga I, von Bahr Greenwood T, et al. Recommendations for the use of etoposide-based therapy and bone marrow transplantation for the treatment of $\mathrm{HLH}$ : consensus statements by the HLH Steering Committee of the Histiocyte Society. J Allergy Clin Immunol Pract. 2018;6(5):1508-1517. doi:10.1016/j.jaip.2018.05.031

12. Yoon JH, Park SS, Jeon YW, et al. Treatment outcomes and prognostic factors in adult patients with secondary hemophagocytic lymphohistiocytosis not associated with malignancy. Haematologica. 2019;104(2):269-276. doi:10.3324/haematol.2018.198655

13. Bergsten E, Horne A, Aricó $M$, et al. Confirmed efficacy of etoposide and dexamethasone in HLH treatment: long-term results of the cooperative HLH-2004 study. Blood. 2017;130(25):2728-2738. doi:10.1182/blood-2017-06-788349

14. Lin TF, Ferlic-Stark LL, Allen CE, Kozinetz CA, McClain $\mathrm{KL}$. Rate of decline of ferritin in patients with hemophagocytic lymphohistiocytosis as a prognostic variable for mortality. Pediatr Blood Cancer. 2011;56(1):154-155. doi:10.1002/pbc.22774

15. Zhou J, Zhou J, Shen DT, Goyal H, Wu ZQ, Xu HG. Development and validation of the prognostic value of ferritin in adult patients with Hemophagocytic Lymphohistiocytosis. Orphanet J Rare Dis. 2020;15(1):71. Published 2020 Mar 12. doi:10.1186/s13023-020-1336-6

16. Locatelli F, Jordan MB, Allen CE, et al. Safety and efficacy of emapalumab in pediatric patients with primary hemophagocytic lymphohistiocytosis. Presented at: American Society of Hematology Annual Meeting, November 29, 2018. Blood. 2018;132(suppl 1):LBA-6. doi:10.1182/blood-2018-120810 